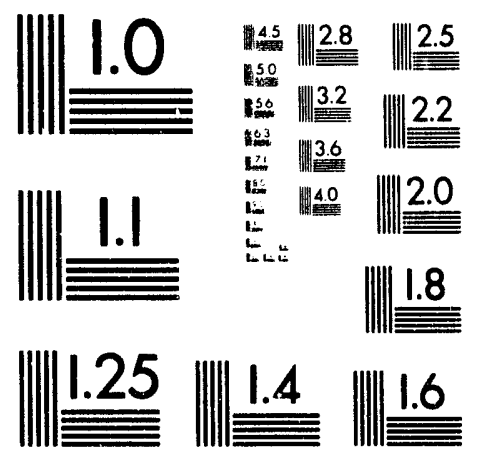



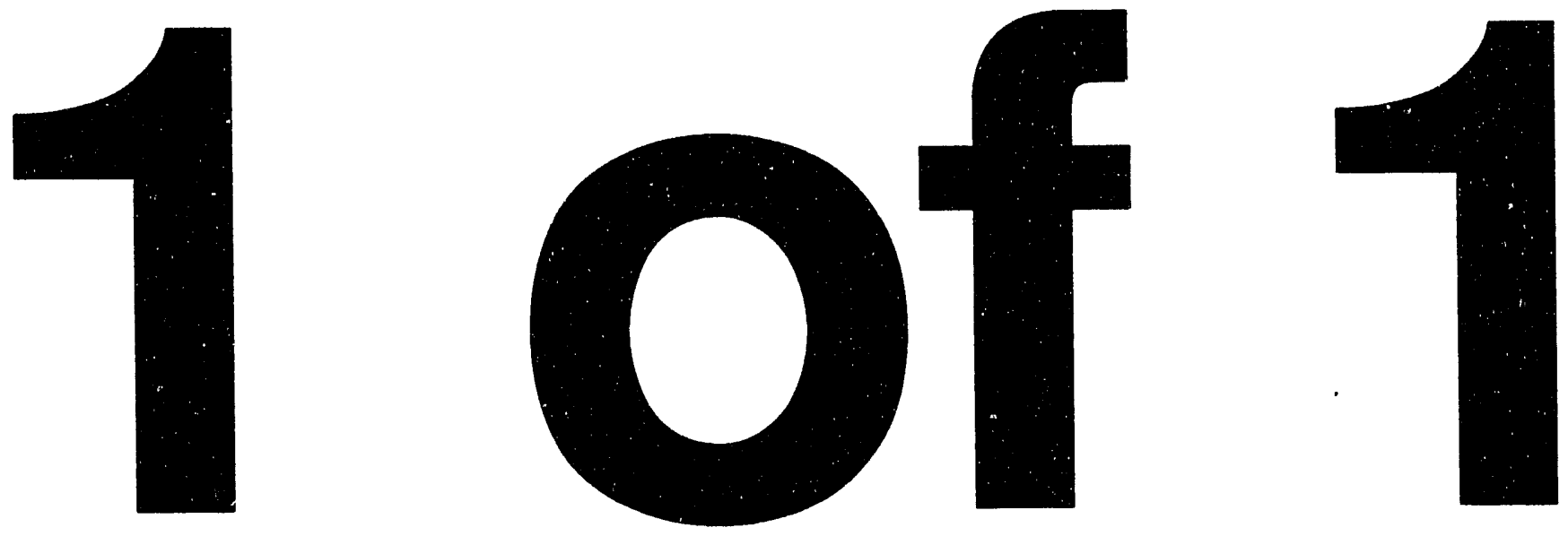


\section{INSTITUTE FOR FUSION STUDIES}

DOE/ET-53088-546

IFSR \#546

On Neutral Plasma Oscillations

B.A. ShadWICK and P.J. Morrison Institute for Fusion Studies The University of Texas at Austin Austin, Texas 78712

June 1993

\section{THE UNIVERSITY OF TEXAS}

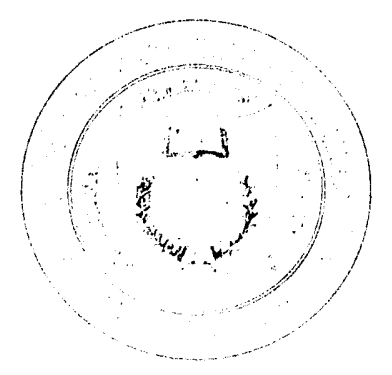

\section{AUSTIN}

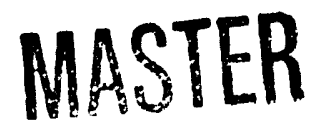

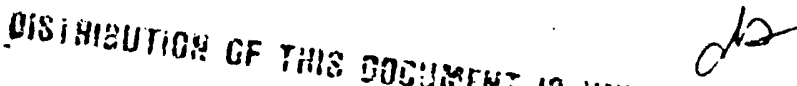




\title{
On Neutral Plasma Oscillations
}

\author{
B.A. Shadwick and P.J. Morrison \\ Institute for Fusion Studies \\ The University of Texas at Austin \\ Austin, Texas 78712
}

\begin{abstract}
We examine the conditions for the existence of spectrally stable neutral modes in a Vlasov-Poisson plasma and show that for stable equilibria of systems that have unbounded spatial domain, the only possible neutral modes are those with phase velocities that correspond to stationary inflection points of the equilibrium distribution function. It is seen that these neutral modes can possess positive or negative free energy.
\end{abstract}

\section{DISCLAIMER}

This report was prepared as an account of work sponsored by an agency of the United States Government. Neither the United States Government nor any agency thereof, nor any of their employees, makes any warranty, express or implied, or assumes any legal liability or responsibility for the accuracy, completeness, or usefulness of any information, apparatus, product, or process disclosed, or represents that its use would not infringe privately owned rights. Reference herein to any specific commercial product, process, or service by trade name, trademark, manufacturer, or otherwise does not necessarily constitute or imply its endorsement, recommendation, or favoring by the United States Government or any agency thereof. The views and opinions of authors expressed herein do not necessarily state or reflect those of the United States Government or any agency thereof. 


\section{Introduction}

In this note we examine the possibility of a Vlasov-Poisson plasma supporting neutral modes (i.e. undamped electrostatic plasma oscillations) and demonstrate the existence of a neutral mode possessing negative free energy. A neutral mode is a mode with a phase velocity, $v_{p}$, that corresponds to a stationary point of the equilibrium distribution function. In spite of the early work by Case ${ }^{1}$ and others, ${ }^{2,3}$ a thorough treatment of neutral oscillations has apparently not been undertaken. Previous analysis of neutral modes has concentrated on modes where the phase velocity of the wave corresponds to a local minima or maxima of the equilibrium distribution. We extend the discussion of neutral modes to the case where $v_{p}$ corresponds to a stationary point of the distribution that is also an inflection point (which we will refer to as a stationary inflection point). We show that stationary inflection point modes are the only neutral modes that can exist in a stable, spatially unbounded plasma.

Neutral modes evidently suffer no Landau damping, a characteristic which makes neutral modes possessing negative free energy of interest from the standpoint of nonlinear instability. Free energy, $\delta^{2} F$, is the energy difference between an equilibrium and a dynamically

accessible perturbed state. ${ }^{4,6}$ Modes that have negative free energy can lead to instability in two ways. Dissipation in a system where the spectrum contains negative energy modes removes energy from these modes resulting in increased amplitude. For example, in finite Larmor radius models dissipation, which has a stabilizing effect on the positive energy mode, leads to instability if the model admits negative energy modes. Negative energy modes can also result in instability through nonlinearities. The prototypical example is the Cherry oscillator. ${ }^{7}$ This system is spectrally stable (i.e. linearly stable in terms of solutions of the form $\left.e^{i \omega t}\right)$ but a resonant nonlinear coupling between the negative and positive energy modes gives rise to solutions that diverge in finite time for arbitrarily small couplings. The nonlinearity 
transfers energy from the negative energy mode to the positive energy mode, causing the amplitude of each to increase catastrophically. Nonresonant nonlinear couplings can also lead to instability. ${ }^{8}$

The remainder of this note is organized as follows. In Sec. II we review the Nyquist method and use it to show that neutral oscillations at stationary inflection points are the only ones allowed for stable equilibria. In Sec. III we derive the free energy of $z$ neutral mode and show that neutral modes are the only modes where the dielectric energy is the correct linear plasma energy. ${ }^{6,12}$ We conclude with a discussion in Sec. IV.

\section{Neutral Modes and Stability}

We consider a one-dimensional Vlasov-Poisson system with an equilibrium electron distribution, $f^{\circ}(v)$, and a fixed, neutralizing ionic background. We assume perturbations of the equilibrium distribution of the form

$$
\delta f(x, v, t)=\iint d \omega d k e^{i(k x-w t)} f_{k}(\omega, v)
$$

and a corresponding electric field perturbation

$$
\delta E(x, t)=\iint d \omega d k e^{i(k x-\omega t)} E_{k}(\omega)
$$

After linearizing, Vlasov's and Poisson's equations become

$$
-i(\omega-k v) f_{k}-\frac{q}{m} E_{k} f^{0}=0
$$

and

$$
i k E_{k}=-4 \pi q \int d v f_{k} .
$$

Combining these equations leads to the plasma dispersion function

$$
\epsilon(k, \omega)=1-\frac{\omega_{p}^{2}}{k^{2}} \int d v \frac{f_{0}^{\prime}}{v-\omega / k}
$$


where $\omega_{p}=4 \pi q^{2} n_{0} / m$ is the electron plasma frequency, $f^{0}=n_{0} f_{0}$ and, in general, the contour is chosen to be that used by Landau in solving the initial value problem. This ensures $\epsilon$ is an analytic function of $\omega$. The dispersion relation $\epsilon=0$ provides the connection between $k$ and $\omega$. For neutral modes, $v_{p}=\omega / k$ is a stationary point of $f_{0}$ and thus the integrand is regular; hence the contour can be chosen simply to be the real axis.

For an equilibrium to be spectrally stable, perturbations must not grow unbounded. This means there must be no solutions of $\epsilon(k, \omega)=0$ with $\operatorname{Im}(\omega)>0$, as such solutions would give rise to exponential growth. We will use the Nyquist method to show that, if the equilibrium distribution is spectrally stable, i.e. $\epsilon(k, \omega)=0$ has no solutions with $\operatorname{Im}(\omega)>0$, neutral modes with phase velocity corresponding to minima or maxima of $f_{0}$ cannot exist. In the following, for the sake ci clarity, we will suppress $k$ in the argument of $\epsilon$.

Consider $^{9}$

$$
\oint_{C} \frac{1}{\epsilon} \frac{\partial \epsilon}{\partial \omega} d \omega .
$$

For any reasonable initial distribution, the only poles in the integrand come from the zeros of $\epsilon$, that is, from the roots of the dispersion relation; the residue at these poles is equal to the multiplicity of the corresponding root. For example, suppose that $\epsilon$ has an $n$-th order root at $\omega=\omega_{0}$. Near $\omega_{0}$,

$$
\epsilon \approx \text { Constant } \times\left(\omega-\omega_{0}\right)^{n}
$$

and

$$
\frac{1}{\epsilon} \frac{\partial \epsilon}{\partial \omega} \approx \frac{n}{\left(\omega-\omega_{0}\right)},
$$

which has a residue of $n$. Thus

$$
N=\frac{1}{2 \pi i} \oint_{C} \frac{1}{\epsilon} \frac{\partial \epsilon}{\partial \omega} d \omega
$$

is the sum of the multiplicities of roots of $\epsilon=0$ enclosed by the contour $C$. If we choose $C$ to consist of a semicircle enclosing the upper half of the $\omega$-plane and the real axis, then $N$ 
will be the number of unstable roots of the dispersion relation. Further, since

$$
\frac{\partial \epsilon}{\partial \omega}=-\frac{\omega_{p}^{2}}{k^{3}} \int d v \frac{f_{0}^{\prime}}{(v-\omega / k)^{2}}
$$

we have

$$
\left|\frac{1}{\epsilon} \frac{\partial \epsilon}{\partial \omega}\right| \rightarrow 0 \quad \text { as } \quad|\omega| \rightarrow \infty
$$

and the contribution to $N$ from circular part of the contour is zero. Therefore

$$
N=\frac{1}{2 \pi i} \int_{-\infty}^{\infty} \frac{\partial \epsilon}{\partial \omega} d \omega=\frac{1}{2 \pi i} \log \left[\frac{\epsilon(\omega=\infty)}{\epsilon(\omega=-\infty)}\right] .
$$

Choosing the phase of $\epsilon$ so that $\epsilon(\omega=-\infty)=1$, we then have $\epsilon(\omega=\infty)=e^{i 2 n \pi}$ and $N=n$. Thus the number of unstable solutions is given by the phase change in $\epsilon$ from $\omega=-\infty$ to $\omega=\infty$. If we think of $\epsilon$ as a curve in the $\omega$-plane, parameterized by $\omega$, then the phase change in $\epsilon$ is given by the number of times $\epsilon(\omega)$ circles the origin as $\omega$ ranges from $-\infty$ to $\infty$. For stability $N$ must be zero for all values of $k^{2}$. To encircle the origin, $\epsilon(\omega)$ must cross the real axis at least once to the left of the origin. Thus we are interested in the sign of $\operatorname{Re}(\epsilon)$ whenever $\epsilon(\omega)$ crosses the real axis. For $\operatorname{Im}(\omega)=0$,

$$
\epsilon_{r} \equiv \operatorname{Re}(\epsilon)=1-\frac{\omega_{p}^{2}}{k^{2}} \int d v \frac{f_{0}^{\prime}}{v-\omega / k}
$$

and

$$
\epsilon_{i} \equiv \operatorname{Im}(c)=-\left.\pi \frac{\omega_{p}^{2}}{k^{2}} f_{0}^{\prime}\right|_{v=\omega / k} .
$$

It is important to remember that, for this analysis, $\omega$ and $k^{2}$ must viewed as independent variables.

Let $v_{\star}$ be the phase velocity of the neutral mode. Now $\epsilon_{r}$ can be rewritten, after integrating by parts as,

$$
\epsilon_{r}=1+\frac{\omega_{p}^{2}}{k^{2}} \int d v \frac{f_{0}\left(v_{\star}\right)-f_{0}(v)}{(v-\omega / k)^{2}}
$$


For the neutral mode, the dispersion relation $\epsilon=0$ gives two equations

$$
\epsilon_{r}=1+\frac{\omega_{p}^{2}}{k^{2}} \int d v \frac{f_{0}\left(v_{\star}\right)-f_{0}(v)}{(v-\omega / k)^{2}} \quad \text { and } \quad \epsilon_{i}\left(v_{\star}\right)=0
$$

that relate $k$ and $\omega$. The second is trivially satisfied since $f_{0}^{\prime}\left(v_{\star}\right)=0$. For the first equation to have a solution,

$$
\int d v \frac{f_{0}\left(v_{\star}\right)-f_{0}(v)}{\left(v-v_{\star}\right)^{2}}<0 ，
$$

since $k^{2}>0$. The central question is: Does the presence of the neutral mode admit unstable solutions of $\epsilon=0$ ? This can be answered by observing that there are two generic possibilities: either $f_{0}^{\prime}$, and consequently $\epsilon_{i}$, changes sign at $v_{\star}$ (i.e. $v_{\star}$ is a maximum or a minimum of $f_{0}$ ) or it does not. Let us consider the first case. Here $\epsilon_{i}$ changes sign as $\omega / k$ passes through $v_{\star}$, that is, the curve $\epsilon(\omega)$ crosses the real axis as $\omega / k$ passes through $v_{\star}$. Further, there is some value of $k=\dot{\kappa}_{\star}$ for which $\epsilon(\omega)$ goes through the origin. Since

$$
\int d v \frac{f_{0}\left(v_{\star}\right)-f_{0}(v)}{\left(v-v_{\star}\right)^{2}}<0
$$

for $k<k_{\star}$ the curve will cross the left of the origin and for $k>k_{\star}$ the curve will cross the right. One of these values of $k$ will result in the curve encircling the origin. Thus for a system with an unbounded spatial domain, it will always be possible to find a value of $k^{2}$ such that $\epsilon(\omega)$ encircles the origin. For an equilibrium distribution where $v_{\star}$ corresponds to a minimum, this is the Penrose criterion. ${ }^{10}$ Hence the existence of this type of neutral mode results in the presence of unstable solutions of the dispersion relation.

The other possibility is that $f_{0}^{\prime}$ does not change sign at $v_{\star}$. This requires that $v_{\star}$ be a zero of $f_{0}^{\prime}$ with even multiplicity. That is, for $v$ near $v_{\star}$,

$$
f_{0}^{\prime} \approx \text { Constant } \times\left(v-v_{\star}\right)^{2 m}
$$

for some $m$. Here, although $\epsilon_{i}\left(v_{\star}\right)=0$, the curve $\epsilon(\omega)$ does not cross the axis, since $f_{0}^{\prime}(v)$ does not change sign as $v$ passes through $v_{\star}$ and the value of $\epsilon_{\uparrow}$ is unimportant. In essence 
the multiple root of $f_{0}^{\prime}$ at $v_{\star}$ prevents $\epsilon(\omega)$ from encircling the origin. Hence $\epsilon=0$ does not admit solutions with $\operatorname{Im}(\omega)>0$. Thus it is possible for neutral modes to exist at such points of $f_{0}$ without losing spectral stability. Further, we see that the only possible neutral modes for a stable equilibrium are modes of this type.

If the system is spatially bounded, the first possibility (i.e. where $v_{\star}$ corresponds to a minima or a maxima of $f_{0}$ ) is not necessarily excluded. Even if there exist exponentially growing solutions of $\epsilon(k, \omega)=0$ for $k<k_{\star}$, it may happen that, the smallest value of $k$, $k_{\min }=k_{\star}$. That is, the neutral mod: has the longest wavelength allowed by the system. Thus no modes with $k<k_{\star}$ exist and the neutral mode is not incompatible with linear stability. For such systems, as shown by Case, ${ }^{1}$ neutral modes with phase velocities corresponding to minima or maxima of the equilibrium distribution are possible. In what follows we will only consider unbounded systems.

\section{Free Energy of Neutral Modes}

Free energy expressions can be obtained from either the Eulerian or Lagrangian variable descriptions. In the Eulerian case, the free energy comes from extremizing the Hamiltonian constrained by certain constants of motion known as Casimirs, which embody conservation of phase space volume. (Unconstrained stationary points of the Hamiltonian correspond to the "vacuum," i.e. where all particles have zero kinetic energy and the perturbed fields are also zero and thus generally uninteresting). This constraint is equivalent to demanding that the perturbation be dynamically accessible, since the Casimirs divide the phase space into constraint surfaces (symplectic leaves) which cannot be crossed by the phase space flow. Here $\delta^{2} F$ is given by ${ }^{4,5}$

$$
\delta^{2} F=\frac{1}{2} \int d x d v\left[H_{0}, g\right]\left[g, f^{0}\right]+\frac{1}{8} \int d x \delta E^{2}[g]
$$


It has the physical interpretation as the energy increase due to such perturbations. This expression is valid for any perturbation arising from a generating function $g$, according to $\delta f=\left[f^{0}, g\right]$, where $f^{0}$ is the equilibrium distribution function and $[$,$] is the usual Poisson$ bracket. The argument of $\delta E$ is shown explicitly in order to emphasize that $\delta E$ is a (known) expression that depends on $\delta f$ and thus $g$ through Poisson's equation. Alternatively, the $\delta^{2} F$ expression can be obtained in the Lagrangian description starting from the Low Lagrangian and restricting to canonical perturbations; i.e., those perturbation derived from a generating function. ${ }^{5}$

Using the linearized Vlasov equation,

$$
f_{k} \equiv i k g_{k} f^{0}=-i E_{k} \frac{q}{m} \frac{f^{0}}{\omega-k v}
$$

the free energy for a single neutral mode with phase velocity $v_{p}$, corresponding to a stationary inflection point of the equilibrium distribution function, is given by

$$
\begin{aligned}
\delta^{2} F & =\frac{1}{8 \pi}\left|E_{k}\right|^{2}-\frac{m}{2} \int d v v\left|g_{k}\right|^{2} k^{2} f^{\prime 0} \\
& =\frac{1}{8 \pi}\left\{\left|E_{k}\right|^{2}-\frac{\omega_{p}^{2}}{k^{2}}\left|E_{k}\right|^{2} \int d v \frac{v f_{0}^{\prime}}{\left(v-v_{p}\right)^{2}}\right\} \\
& =\frac{1}{8 \pi}\left|E_{k}\right|^{2} l\left\{(\therefore, \omega)-v_{p} \frac{\omega_{p}^{2}}{k^{2}} \int d v \frac{f_{0}^{\prime}}{\left(v-v_{p}\right)^{2}}\right\} .
\end{aligned}
$$

Since $\epsilon(k, \omega)=0$

$$
\delta^{2} F=-\frac{1}{8 \pi}\left|E_{k}\right|^{2} v_{p} \frac{\omega_{p}^{2}}{k^{2}} \int d v \frac{f_{0}^{\prime}}{\left(v_{p}-v\right)^{2}} .
$$

For the distributions that we are considering, $f_{0}^{\prime}\left(v_{p}\right)=0$, thus the expression for $\epsilon$ can be safely differentiated with respect to $\omega$, from which we find

$$
\delta^{2} F=\frac{1}{8 \pi}\left|E_{k}\right|^{2} \omega \frac{\partial \epsilon}{\partial \omega}
$$

It is important to remember that in the above, $v_{p}$ is the phase velocity in the frame where the energy is a minimum, i.e. in the center of mass frame. Note that the integrals are frame 
independent: in a frame moving with velocity $V$ with respect to the original frame, the new velocity, $\tilde{v}$, is

$$
\tilde{v}=v-V, \quad \text { and } \quad \tilde{f}_{0}(\tilde{v})=f_{0}(v-V)
$$

giving

$$
\int d v \frac{f_{0}^{\prime}(v)}{\left(v_{p}-v\right)^{2}} \rightarrow \int d \tilde{v} \frac{f_{0}^{\prime}(v-V)}{\left(\tilde{v}_{p}-\tilde{v}\right)^{2}},
$$

where $\tilde{v}_{p}=v_{p}+V$ is the Doppler shifted phase velocity. ${ }^{11}$ If the variable of integration is shifted, $\tilde{v} \rightarrow \tilde{v}-V$, we obtain the same expression as in the original frame. Thus the free energy depends on the frame only through the phase velocity, i.e. $\delta^{2} F=-v_{p} \times$ constant and $\delta^{2} \tilde{F}=\tilde{v}_{p} / v_{p} \delta^{2} F$. The sign of $\delta^{2} F$ is determined by

$$
-v_{p} \int d v \frac{f_{0}^{\prime}}{\left(v_{p}-v\right)^{2}}
$$

and so depends on the precise shape of $f_{0}$.

Consider an equilibrium distribution with a single maximum which supports a neutral mode with phase velocity $v_{p}$. In some frame

$$
f_{0}^{\prime}=-v\left(v-v_{p}\right)^{2} P(v),
$$

where $P(v) \geq 0$ for all $v$. While this may not be the center of mass frame, the existence of the mode is frame independent. Let $P_{n}$ denote the $n$-th moment of $P$. Cleariy

$$
P_{2 n}>0 \text {. }
$$

The wavenumber of the neutral mode is given by $\epsilon_{\mathrm{r}}=0$, namely

$$
\begin{aligned}
k^{2} & =-\omega_{p}^{2} \int d v \frac{f_{0}^{\ell}}{v-v_{p}} \\
& =\omega_{p}^{2} \int d v\left(v-v_{p}\right) P(v) \\
& =\omega_{p}^{2}\left(v_{p} P_{1}-P_{2}\right) .
\end{aligned}
$$


For the mode to exist, $k^{2}>0$ which means $v_{p} P_{1}>0$ since $P_{2}>0$. In the chosen frame

$$
\begin{gathered}
\delta^{2} F \propto v_{p} \int d v v P(v) \\
\quad=v_{p} P_{1}>0 .
\end{gathered}
$$

The velocity of the center of mass of the plasma, $v_{\mathrm{cm}}$, is given by

$$
\begin{aligned}
v_{\mathrm{cm}} & =\frac{1}{n_{0}} \int d v v f^{0}=-\frac{1}{2} \int d v v^{2} f_{0}^{\prime} \\
& =\frac{1}{2} v_{r}^{2} P_{3}-v_{p} P_{4}+\frac{1}{2} P_{5} .
\end{aligned}
$$

The requirements that the distribution function be normalized and vanish at infinity constrains the first and third moments of $P$ :

$$
P_{1}=\frac{1}{2 v_{p}^{3}}\left(1+3 v_{p}^{3} P_{2}-P_{4}\right)
$$

and

$$
P_{3}=\frac{1}{2 v_{p}}\left(v_{p}^{2} P_{2}+P_{4}-1\right)
$$

Using this in the expressions for $k^{2}$ and $v_{\mathrm{cm}}$ gives

$$
\begin{aligned}
& k^{2}=\frac{\omega_{p}^{2}}{2 v_{p}^{2}}\left(1+v_{p}^{2} P_{2}-P_{4}\right) \\
& v_{\mathrm{cm}}=\frac{v_{p}}{4}\left(v_{p}^{2} P_{2}-3 P_{4}-1\right)+\frac{1}{2} P_{5} .
\end{aligned}
$$

Transforming to the center of mass frame, the phase velocity of the neutral mode becomes

$$
\tilde{v}_{p}=\frac{v_{p}}{4}\left(3+v_{p}^{2} P_{2}-3 P_{4}\right)+\frac{1}{2} P_{5}
$$

and

$$
\delta^{2} \tilde{F} \propto \frac{v_{p} P_{1}}{4}\left(3+v_{p}^{2} P_{2}-3 P_{4}+2 \frac{P_{5}}{v_{p}}\right)
$$


Since these moments of $P$ are arbitrary, the free energy in the center of mass frame can be either positive, negative or zero depending on $f_{0}$.

We now specialize to the case where equilibrium distribution is symmetric in some frame. In that frame (also the center of mass frame) $f_{0}^{\prime}$ has the form

$$
f_{0}^{\prime}=-v\left(v^{2}-v_{p}^{2}\right)^{2} P(v)
$$

where $P(v)$ is a positive definite, symmetric function. Here

$$
P_{2 n}>0 \quad \text { and } \quad P_{2 n+1}=0
$$

giving

$$
k^{2}=\omega_{p}^{2}\left(v_{p}^{2} P_{2}-P_{1}\right)
$$

and

$$
\delta^{2} F \propto 2 v_{p}^{2} P_{2}>0
$$

For this class of distributions, all neutral modes have positive free energy.

We see that of the distributions that nave a single maximum, those that are symmetric can only support neutral modes with positive free energy, while thooe that are nonsymmetric allow for neutral modes to have negative free energy. Further, for a symmetric distribution with more than one maximum, $P_{2}$ and $P_{1}$ are not necessarily positive (since $P$ is no longer positive definite) thus admitting the possibility of the neutral mode having negative free energy. 12

\section{Discussion}

Previous work on neutral modes, Case ${ }^{1}$ ("Class $1 c^{n}$ ), only considered modes that correspond to either minima or maxima of the equilibrium distribution. Such modes can only be supported by a linearly stable equilibrium if the spatial extent of the system is such that $k_{\min }$ is 
sufficiently large so that $\epsilon_{r}\left(\omega / k_{\min }=v_{*}\right)>0$. Stationary inflection point modes are the only linear undamped modes that can be supported by a spatially unbounded, stable equilibrium. Furthermore, we have shown that, only for neutral modes is

$$
\frac{1}{8 \pi}\left|E_{k}\right|^{2} \omega \frac{\partial \epsilon}{\partial \omega}
$$

the correct expression for the free energy. ${ }^{12}$ This energy expression is correct both for inflection points modes as well as the neutral modes of the type studied by Case. (The correct energy of perturbations about stable equilibria that do not support neutral modes is not the above but is given by an expression derived in Ref. 6.)

The nonlinear stability of neutral modes has previously been examined. ${ }^{13}$ Unfortunately, this analysis did not take into account that neutral modes of the type described here exist in linear theory and that the condition $k^{2}>0$ is necessary for their existence. The case of nonlinear undamped plasma waves has been recently explored both numerically ${ }^{14}$ and analytically ${ }^{15}$ for spatially nonhomogeneous equilibria. Inflection point neutral modes seem to be a likely candidate for the linear limit of these nonlinear undamped waves in the sense that a homogeneous equilibrium obtained by spatial averaging supports inflection point modes with the same phase velocity as the observed nonlinear oscillations.

In a model that includes trapped particles, BGK modes with phase velocities corresponding to the trapped particles become van Kampen modes in the limit that the trapped particle density goes to zero, provided that the equilibrium distribution function has a discontinuity. ${ }^{16}$ If $f^{0}$ is smooth then instead of a van Kampen mode, one obtains a quasimode, ${ }^{17}$ which is subject to Landau damping. If $f^{0}$ has a stationary inflection point then in this limit one obtains a neutral mode. The neutral eigenmode limit seems to be a natural counterpart to the van Kampen (singular) eigenmode for the case of continuous $f^{\circ}$ in that both are true eigenmodes and persist for all time. 


\section{Acknowledgments}

The work was supported by the U.S. Department of Energy contract \#DE-FG05-80ET53088. 


\section{References}

${ }^{1}$ K.M. Case, Ann. Phys. (N.Y.) 7, 349 (1959); K.M. Case, Phys. Fluids 21, 249 (1978).

${ }^{2}$ M.D. Arthur, William Greenberg, and P.F. Zweifel, Phys. Fluids 20, 1296 (1977).

${ }^{3}$ C.E. Siewert, J. Math. Phys. 18, 2509 (1977).

'P.J. Morrison and M. Kotschenreuther, in Proceedings of the 4th International Workshop on Nonlinear and Turbulent Processes in Physics, Kiev, USSR, 1989 (World Scientific); M. Kotschenreuther, in Plasma Physics and Controlled Nuclear Fusion Research 1986 (IAEA, Vienna, 1978), Vol. 2, p. 149.

${ }^{5}$ P.J. Morrison and D. Pfirsch, Phys. Fluids B 2, 1105 (1990); P.J. Morrison, and D. Pfirsch, Phys. Rev. A 40, 3898 (1989).

${ }^{6}$ P.J. Morrison and D. Pfirsch, Phys. Fluids B 4, 3038 (1992).

${ }^{7}$ T.M. Cherry, Trans. Cambridge Philos. Soc. 23, 199 (1925).

${ }^{8}$ C.S. Kuney, Nonlinear Instability and Chaos in I'lasma Wave-Wave Interactions, $\mathrm{Ph} . \mathrm{D}$. Dissertation, The University of Texas at Austin, (1993). Available as IFS Report \#588, The Institute for Fusion Studies, The University of Texas at Austin, Austin, Texas 78712.

${ }^{9}$ N.A. Krall and A.W. Trivelpiece Principles of Plasma Physics (McGraw-Hill, New York, 1973), Sec. 9.6, p. 464.

${ }^{10}$ O. Penrose, Phys. Fluids 3, 258 (1960).

${ }^{11}$ J. Dawson, Phys. Fluids 4, 869 (1961).

${ }^{12}$ B.A. Shadwick and P.J. Morrison, Bull. Am. Phys. Soc. 36, 2329 (1991). 
${ }^{13}$ A. Simon, M.N. Rosenbluth, Phys. Fluids 19, 1567 (1976).

${ }^{14}$ L. Demeio and J.P. Holloway, J. Plasma Phys. 46, 63 (1991).

${ }^{15}$ J.P. Holloway and J.J. Dorning, Phys. Rev. A 44, 3856 (1991).

${ }^{16}$ I.B. Bernstein, J.M. Greene, M.D. Kruskal, Phys. Rev. 108, 546 (1957).

${ }^{17}$ H. Schamel, J. Plasma Phys. 13, 139 (1975); H. Schamel, Physica Scripta 20, 336 (1979). 

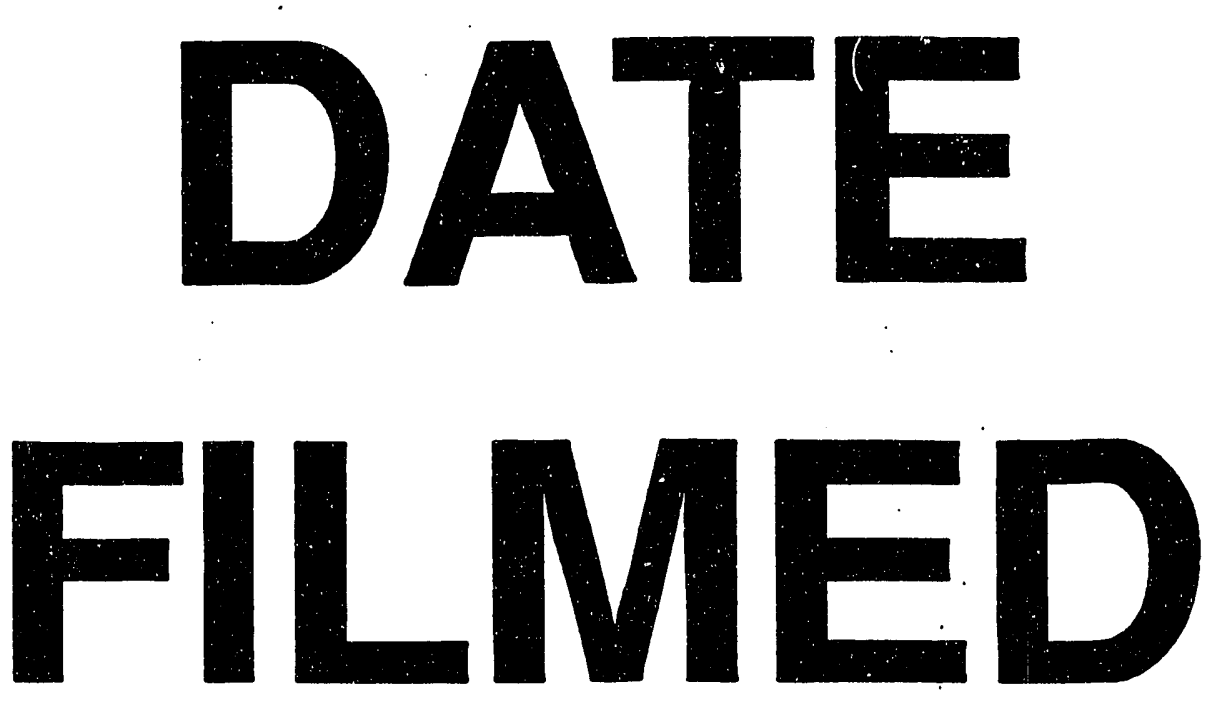

$10 / 13 / 93$
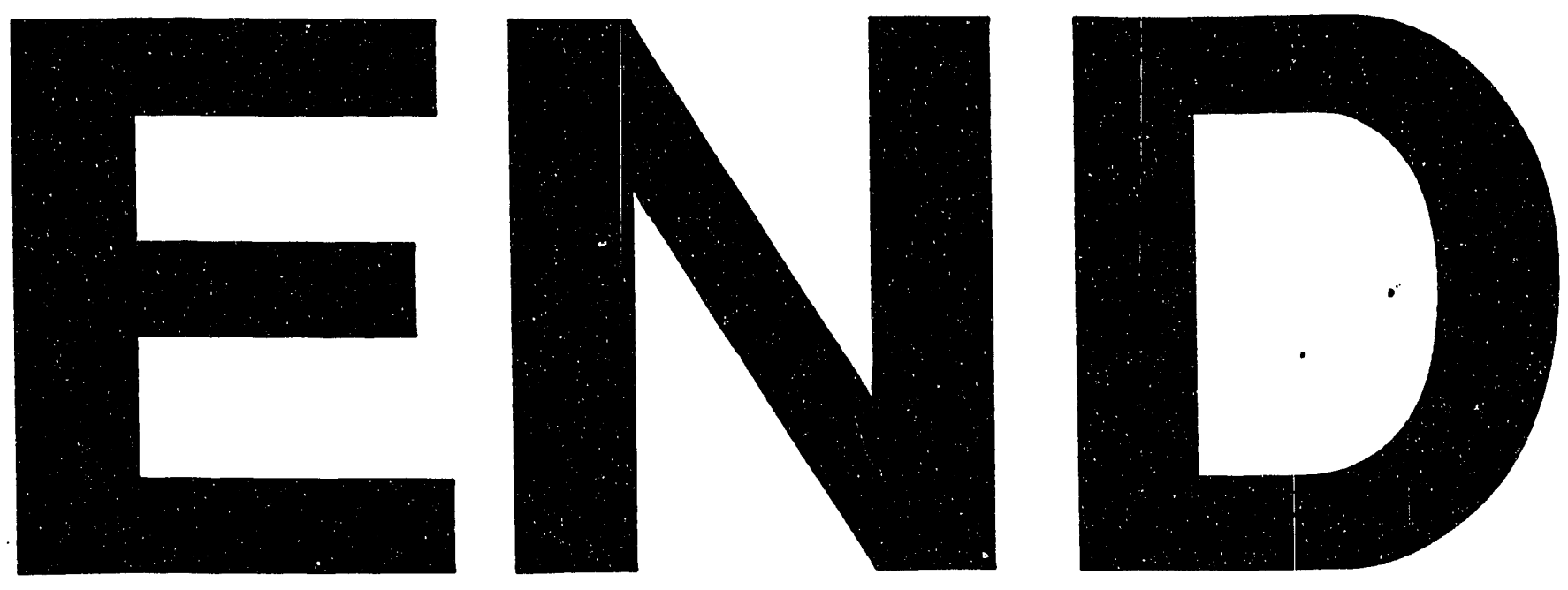
